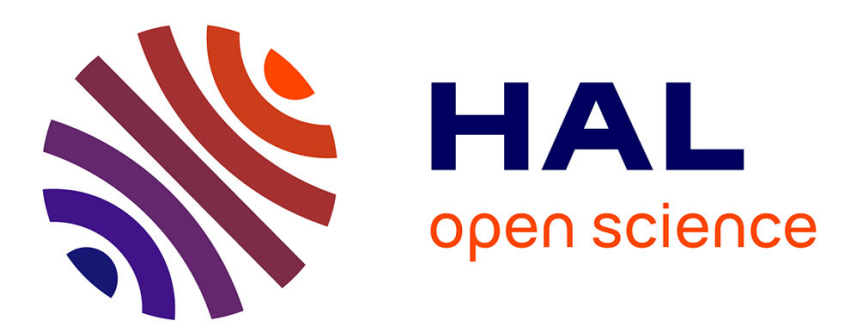

\title{
De la condamnation de la mixité à la crainte de l'altération. Où sont les femmes dans la haine du théâtre?
}

Sarah Nancy

\section{- To cite this version:}

Sarah Nancy. De la condamnation de la mixité à la crainte de l'altération. Où sont les femmes dans la haine du théâtre?. Littératures classiques, 2019, La haine du théâtre. Controverses européennes sur le spectacle, Vol. 2: Discours et arguments. hal-03380979

\section{HAL Id: hal-03380979 \\ https://hal.science/hal-03380979}

Submitted on 18 Oct 2021

HAL is a multi-disciplinary open access archive for the deposit and dissemination of scientific research documents, whether they are published or not. The documents may come from teaching and research institutions in France or abroad, or from public or private research centers.
L'archive ouverte pluridisciplinaire HAL, est destinée au dépôt et à la diffusion de documents scientifiques de niveau recherche, publiés ou non, émanant des établissements d'enseignement et de recherche français ou étrangers, des laboratoires publics ou privés. 


\section{littératures classiques}

La haine du théâtre.

Controverses européennes

sur le spectacle

Vol. 2 : Discours et arguments

$N^{\circ} 99 / 2019$

PRESSES UNIVERSITAIRES DU MIDI 


\section{De la condamnation de la mixité à la crainte de l'altération Où sont les femmes dans la haine du théâtre?}

Sur le terrain bien connu des querelles sur la moralité du théâtre en France dans la deuxième moitié du XVII ${ }^{e}$ siècle ${ }^{1}$, c'est à un motif lui aussi bien connu que nous nous intéressons : celui des femmes, ou du féminin. Motif bien connu sur un terrain bien connu : c'est peut-être le problème. Le motif adhère si bien aux discours hostiles au théâtre qu'il semble difficile d'en dire quelque chose de spécifique : soit il est englobé dans une guerre générale contre le plaisir - voir Jonas Barish dans The Antitheatrical Prejudice ${ }^{2}$ ou bien Georgia Cowart étudiant la façon dont l'opéra, après 1694, est agrégé au théâtre dans les textes de condamnation $^{3}-$, soit sa rencontre avec le genre dramatique est considérée comme fortuite - Laurent Thirouin estime par exemple que ce motif, qui relève d'une "prévention [...] à l'égard de la mixité » des plus "précaire[s] », n'a en fait aucun rapport précis avec le problème du théâtre ${ }^{4}$. Sans doute parce qu'il

1 Dans la deuxième moitié du siècle se situent ce que Laurent Thirouin repère comme les deuxième et troisième grands moments de la querelle : celui des années 1660-1670 et celui qui suit la publication de la lettre du P. Caffaro en 1694. Ces moments ont pour particularité de mettre en cause non plus les dérives possibles du genre théâtral, mais bien le genre même, autour de textes puissants et érudits. Voir L. Thirouin, L'Aveuglement salutaire. Le réquisitoire contre le théâtre dans la France classique, Paris, H. Champion, 1997, p. 17-18.

2 J. Barish, The Antitheatrical Prejudice, Berkeley / Los Angeles / Londres, University of California Press, 1981. J. Barish y insiste sur ce qui sépare la conception française de celle des néoplatonistes italiens: chez les Français, ce préjugé antithéâtral «va de pair avec un antiféminisme, qui, plus largement, participe d'une guerre contre le plaisir » [antitheatricalism joins hands with antifeminism as part of the war against pleasure] » (p. 203).

3 G. Cowart, "Of women, sex and folly: Opera under the Old Regime », Cambridge Opera Journal, vol. 6, n 3, nov. 1994, p. 205-220.

4 «Que cessent les préventions à l'égard de la mixité, que les contacts entre les hommes et les femmes ne soient plus assimilés à l'impureté, et ce discours sur le théâtre se trouve privé de sa consistance » (L. Thirouin, op. cit., p. 183-184). 
nous place sur le terrain des sentiments - parler de haine n'est pas simplement parler de querelle - la question de la « haine du théâtre » invite à reprendre le dossier. Où sont les femmes dans la haine du théâtre ? Pourquoi et à quel titre sont-elles un motif moteur dans la condamnation du genre?

Ces questions nous guideront dans l'examen des arguments des détracteurs du théâtre à la fin du XVIIe siècle pour organiser ceux-là en fonction de la manière dont ils convoquent les femmes réelles, c'est-à-dire les femmes susceptibles d'être concrètement concernées par le théâtre, ou dont ils s'en éloignent, au contraire. Car comme l'envisage Pierre Nicole dès le premier paragraphe de son traité, le rapport des femmes avec le théâtre semble ne pas se réduire à leur présence :

On ne parle pas seulement des dérèglements grossiers, et de la manière dissolue dont les femmes y paraissent, parce que ceux qui justifient la Comédie en séparent toujours ces sortes de désordres par l'imagination, quoiqu'on ne les en sépare jamais effectivement. ${ }^{5}$

Notre ambition sera donc de comprendre non seulement ce qui, dans la haine du théâtre, tient à la possibilité de la présence des femmes, à la «manière » dont elles «paraissent», mais aussi de considérer cette résistance « effective » à une exclusion théorique ("par l'imagination») dont parle Nicole - autrement dit, de s'interroger sur ce qui, des «désordres » liés aux femmes, semble indépendant des circonstances et donc consubstantiel au genre théâtral.

\section{La présence mise en cause : mixité, débauche}

Parmi les arguments incriminant le plus concrètement les femmes, pour accabler le genre dramatique, il y a celui de leur présence avec les hommes dans les lieux du théâtre. C'est l'argument de la mixité. Pour Tertullien - on sait combien cette référence est centrale, puisqu'elle pallie en quelque sorte le défaut de sources dans l'Écriture sainte au sujet du théâtre ${ }^{6}-$, le théâtre doit son impudicité au double patronage de Vénus et de Liber ${ }^{7}$ : il constitue une occasion de débauche parce qu'il rassemble dans la salle hommes et femmes qui cherchent à se montrer et nouent des complicités vicieuses à travers les discussions passionnées au sujet des comédiens ${ }^{8}$. Le janséniste Alexandre Varet s'en souvient dans son « Avis sur la comédie » (1666) :

5 P. Nicole, Traité sur la Comédie et autres pièces d'un procès du théâtre, éd. L. Thirouin, Paris, H. Champion, 1998, p. 36.

6 Tertullien, qui avait affronté d'emblée ce problème dans De spectaculis, devient lui-même, logiquement, une autorité en matière de combat contre le théâtre : voir L. Thirouin, op. cit., p. 29.

7 Tertullien, Les Spectacles, X, 7-8, éd. M. Turcan, Paris, Les Éditions du Cerf, 1986, p. 189-191.

$8 \quad$ Ibid., XXV, 2, p. 287. 
Ge que Tertullien a estimé être «le plus grand scandale» qui se trouvait dans les spectacles des païens, ne se rencontre-t-il pas dans les comédies? Les hommes et les femmes, les jeunes gens et les jeunes filles, ne s'y trouvent-ils pas ensemble, et n'y vontils pas avec tout l'ajustement et l'agrément qui leur est possible ?9

Dans le dossier patristique qui accompagne son traité, paru la même année que celui de Varet, le prince de Conti cite Clément d'Alexandrie : c'est sensiblement dans les mêmes termes que celui-ci articule la condamnation des assemblées théâtrales à celle de la «confusion » et de l'«impureté » qu'occasionne la rencontre entre les sexes ${ }^{10}$. Et chez Joseph de Voisin, ancien aumônier du prince de Conti, dans sa volumineuse et virulente réfutation de la Dissertation sur la condamnation des théâtres de l'abbé d'Aubignac ${ }^{11}$, c'est l'autorité tridentine de saint Charles Borromée qu'on trouve à l'appui de la condamnation du «mélange qui se fait dans le bal, et à la Comédie, d'hommes et des femmes" ${ }^{12}$.

Risque d'être «ensemble », «mélange », « confusion»: la présence des femmes est donc en cause de manière fondamentale dans la condamnation du théâtre. C'est leur rencontre avec les hommes, et le risque de séduction mutuelle qui en découle - où l'on comprend qu'il n'y a pas de séduction passive, et qu'on est aussi coupable de plaire que de chercher à plaire ${ }^{13}$ - qui fait du théâtre un lieu à haïr. Parmi les femmes, certaines sont bien sûr particulièrement dangereuses : celles qui se produisent sur scène. Même si Victoria Scott a pu montrer qu'il fallait attendre le XVIIIe siècle pour que se déploie pleinement une condamnation des comédiennes ${ }^{14}$, les textes du siècle précédent n'omettent pas d'en signaler le danger. Voisin écrit :

on ne peut dire ni s'imaginer sans ignorance, ou sans une témérité présomptueuse, que le Théâtre où l'on voit des femmes qui jouent des Comédies, et d'autres qui y assistent,

9 A. Varet, "Avis touchant la comédie », De l'Éducation chrétienne des enfants, selon les maximes de l'Écriture Sainte et les instructions des Saints Pères de l'Église, Paris, P. Promé, 1666, p. 284-285.

10 «On peut justement appeler les théâtres et la carrière des courses publiques une chaire de pestilence. Car tout ce qui se fait en ces lieux est plein de confusion et d'iniquité. Ces assemblées ne fournissent que trop de sujets d'impureté, où les hommes et les femmes étant ensemble, s'occupent à se regarder. C'est là où se tiennent de pernicieux conseils, lorsque les regards lascifs excitent de mauvais désirs » (Clément d'Alexandrie, Le Pédagogue, 1.3, c.11, cité et traduit par A. de Conti dans «Sentiments des Pères de l'Église sur la Comédie et sur les spectacles », Traité de la Comédie et des spectacles ; cité par L. Thirouin, op. cit., p. 183).

11 Fr. Hédelin, abbé d'Aubignac, Dissertation sur la condamnation des théâtres, Paris, N. Pépingué, 1666.

12 Charles Borromée, Traité contre les danses et les comédies; cité par J. de Voisin, La Défense du traité de Monseigneur le Prince de Conti touchant la comédie et les spectacles, ou la réfutation d'un livre intitulé Dissertation sur la condamnation des théâtres, Paris, L. Billaine, 1671, p. 386.

13 Voir, à ce sujet, H. Merlin-Kajman, "Les troubles du masculin en France au XVII ${ }^{\mathrm{e}}$ siècle ", dans H. Amigorena et F. Monneyron(dir.), Le Masculin. Identité, fictions, dissémination, Paris, L'Harmattan, 1998, p. 11-57.

14 V. Scott, «The Actresses and Utopian Theatre Reform in Eighteenth-Century France ", Theatre Research International, vol. 7, $\mathrm{n}^{\circ} 1$, mars 2002, p. 18-27. 
n'est point un lieu dangereux. Il est indubitable qu'une Comédienne galante, et de bonne mine, ressemble le serpent nommé Scitale qui selon Solin, et S. Isidore, est si beau, et a des écailles émaillées d'or, et de diverses couleurs si vives, et si reluisantes, qu'il ravit le cœur et l'affection de celui qui regarde. ${ }^{15}$

Deux catégories de femmes, donc, font valoir la nocivité du théâtre : celles qui « assistent » au spectacle et celles qui y «jouent »- et ce sont ces dernières qui sont comparées au serpent légendaire, comme le seront quelques années plus tard les actrices de l'opéra, « ces basilics qui empoisonnent et tuent les âmes par les oreilles ${ }^{16} »$.

Par leurs riches connotations - séduction maléfique, incitation au vice -, ces images de serpent renvoient à une autre circonstance concrète déjà invoquée par Tertullien: le métier de comédienne serait indissociable des mœurs déréglées, voire de la prostitution supposée se pratiquer dans les théâtres. On note d'ailleurs que Scudéry, dans sa Comédie des comédiens, avait déjà dû se défendre de cette accusation par le biais du personnage de la Beau Soleil ${ }^{17}$.

\section{La représentation mise en cause : contagion, effémination, corruption}

La tentation physique que représentent les femmes dans la salle fait donc partie des arguments les plus concrets qui alimentent la haine du théâtre. Mais tout le danger ne réside pas dans les corps réunis. Le théâtre, au-delà même de la possibilité qu'on lui prête de fournir l'occasion réelle de rapports avec les femmes, donne à voir ces rapports : la Comédie est le lieu où l'on « on [ne] représente que des galanteries ${ }^{18}$ », et où « cet aveu, dont on rougit dans le secret, est jugé digne d'être révélé au public ${ }^{19} »$. L'accusation est omniprésente et s'appuie sur l'autorité d'Augustin. Le rapprochement structurel que fait celui-ci entre tragédie et passion amoureuse est en effet repris de manière simplifiée ${ }^{20}$ comme un lien de cause à effet : parce qu'il représente la passion amoureuse, le théâtre est nocif.

15 J. de Voisin, La Défense du traité de Monseigneur le Prince de Conti, op. cit., p. 408. Il se réfère au jésuite P. de Guzman.

16 L.-F. Ladvocat, Description de la vie et meurs [...] des filles de l'Opéra, éd. J. de La Gorce, s.l., Cicero, 1993, p. 77.

17 G. de Scudéry, La Comédie des Comédiens, éd. J. Crow, Exeter, University of Exeter, 1975, p. 11.

18 P. Nicole, op. cit., § XXII, p. 84.

19 J.-B. Bossuet, «Lettre de Bossuet au P. Caffaro, théatin », Maximes et réflexions sur la Comédie, éd. A. Gazier, Paris, Belin, 1881, p. 6.

20 Dans les chap. 1 et 2 du livre III des Confessions, saint Augustin établit un rapport structurel entre passion amoureuse et tragédie : dans les deux cas, le mouvement vers autrui (amour, pitié) n'est qu'un détournement de la bienveillance et du sentiment naturel de l'amour du prochain : voir L. Thirouin, "La condamnation morale du théâtre. L'autorité de saint Augustin ", dans Religion et politique: les avatars de l'augustinisme, Saint-Étienne, Publications de l'Université de Saint-Étienne, 1998, p. 275-296. 
Ce danger de la représentation du rapport entre les sexes est ainsi l'occasion d'un autre type de mise en cause de la présence des femmes au théâtre - non, cette fois, en raison de leur interaction directe avec les hommes lors du spectacle, mais en raison de la grande réceptivité au spectacle qu'on leur prête. Bossuet déplore qu'à la comédie une jeune fille puisse entendre «les discours où une personne de son sexe parle de ses combats, où elle avoue sa défaite, et l'avoue à son vainqueur même ${ }^{21} »$. Et le problème n'est pas seulement que se perfectionne ainsi l'art de "faire l'amour », comme le dénonce déjà Francesco Maria del Monaco dans son traité de $1630^{22}$, mais que les femmes développent des attentes irréalistes qui les rendent ensuite inaptes à bien remplir leur rôle dans la société. Il est remarquable que ce scénario constitue une version inversée de celle de saint Jean Chrysostome, qui a pu en fournir l'inspiration, laquelle met en scène non pas une femme mais un homme distrait de ses devoirs ${ }^{23}$. En voici la réécriture par Voisin, qui s’inspire lui-même de la version de Nicole en la complétant et, du même coup, en radicalisant la responsabilité des femmes :

Les femmes voyant sur le théâtre les adorations qu'on y rend à celles de leur sexe, dont elles voient l'image, et la pratique dans les compagnies de divertissement, où de jeunes gens leur débitent ce qu'ils ont appris dans les Comédies, et les traitent en nymphes, et en Déesses, s'impriment tellement dans la fantaisie cette sorte de vie, [emprunt au §XXII du Traité sur la Comédie] qu'elles sont bien aises de tenir dans le cœur des hommes une place qui n'appartient qu'à Dieu seul, en prenant plaisir d'être l'objet de leurs passions [ ...]. Et comme elles ne s'occupent que des galanteries, elles prennent insensiblement une disposition d'esprit toute romanesque, qui les dégoûte tellement de toutes les actions sérieuses, que les petites affaires de leur ménage leur deviennent insupportables : et quand elles reviennent dans leurs maisons avec cet esprit évaporé, et tout plein de ces folies, elles y trouvent tout désagréable, et surtout leurs maris, qui étant occupés de leurs affaires, ne sont pas toujours en humeur de leur rendre des complaisances ridicules, qu'on rend aux femmes dans les Comédies [emprunt encore au $\S$ XXII] [...].24

Avec ce scénario d'une pédagogie vicieuse du théâtre, c'est donc bien encore la présence des femmes qui est incriminée, mais dans une temporalité différée. Ge qui inquiète, c'est la manière dont elles exportent le principe du théâtre hors les murs pour introduire dans la société un ferment de division. On voit donc s'ajouter au danger de la présence un danger en un sens moins concret : celui de

21 J.-B. Bossuet, op. cit., p. 6.

22 «Là les jeunes gens se corrompent, les filles se familiarisent avec l'amour profane, dont ils entendent si agréablement parler»(Fr. M. Del Monaco, In Actores et Spectatores Comædiarum nostris temporis parenesis, Padoue, L. Pasquato, 1630, cité par A. Lalouette, Histoire et abrégé des ouvrages latins, italiens et français, pour et contre la comédie et l'opera, Paris, C. Robustel, 1697, p. 24).

23 Jean Chrysostome, Homélie contre les jeux du cirque et des théâtres, Euvres complètes, éd. et trad. J.-Fr. Bareille, Paris, L. Vivès, 1868, t. V, p. 599. Nous remercions Guillaume Navaud de nous avoir indiqué cette référence.

24 J. de Voisin, op. cit., p. 347. 
la circulation nocive des objets représentés en raison de la vulnérabilité des femmes.

Et de fait, cette prétendue vulnérabilité introduit à un autre niveau de fonctionnement de la référence au féminin qui, sans le congédier, transpose le problème de la présence des femmes en une opération plus abstraite, du moins pouvant s'effectuer dans d'autres corps que les leurs : parce que les femmes sont réputées vulnérables, on qualifie d' "effémination » l'effet d'emprise du théâtre sur les hommes qui participent ou assistent à la représentation. L'accusation d'effémination des comédiens n'est pas neuve, et c'est d'ailleurs en remontant cette histoire que d'Aubignac cherche à sauver les comédiens ${ }^{25}$ : tous ne sont pas des histrions et tous ne sont pas efféminés, allègue-t-il. Mais Voisin répond que la catégorie des comédiens n'est pas divisible, qu'elle est en elle-même efféminante. Pour l'affirmer, il change d'échelle en invoquant l'accusation qu'adresse Tacite à l'institution des «jeux quinquennaux», celle d'avoir efféminé la rhétorique :

On disait que [...] [la] jeunesse se laissait aller peu à peu à l'oisiveté des Grecs, et prenait leurs plaisirs, leurs exercices et leurs sales amours, [...] : et qu'il ne leur restait plus qu'à descendre tout nus en l'arène, et de prendre le Ceste au lieu de la cuirasse et de l'épée. Que les Augures n'apprendraient pas à vivre saintement, et les Chevaliers à devenirs bons juges, en ne s'étudiant qu'à savoir toute la mollesse des tons, et des nombres de la Musique. 26

Citer ce raisonnement par lequel Tacite fait déteindre sur les jeux l'identité altérée des Grecs (avec allusion à «leurs sales amours ») permet à Voisin de montrer que le théâtre n'est pas seulement une pratique aux conséquences éventuellement efféminantes, mais un principe fondamental de diminution de la virilité$^{27}$. Un tel réflexe est significatif : l'effémination est du côté de ce féminin indissociable du théâtre quand bien même les femmes réelles ne seraient pas en jeu. En cela, le raisonnement que tient René Rapin dans ses Réflexions sur la poétique de ce temps (1674) pour circonscrire historiquement l'effémination provoquée par le théâtre est tout aussi irrecevable par les détracteurs du genre: expliquer que le théâtre moderne a fait l'erreur de s'orienter vers la « galanterie » et les «tendresses outrées » par désir de " plaire davantage aux femmes ${ }^{28}$ », c'est accabler le fonctionnement d'un genre qui se nourrit des attentes de l'assistance, c'est-à-dire, selon une vision rigoriste, qui s'ajuste à la faiblesse humaine. Bernard Lamy, dans ses Nouvelles réflexions sur l'art poétique, est très clair à ce sujet : « tout ce plaisir [...] ne vient que de notre corruption, qui nous fait trouver du

25 Fr. Hédelin, abbé d'Aubignac, op. cit., p. 150-151, 201, 215 et 223.

26 Tacite, Annales, XIV, 20, cité par J. de Voisin, op. cit., p. 34-35.

27 Sur cette obsession de l'effémination, voir aussi J. de Voisin, op. cit., p. 164, 165, 199, 200, 202, 210, 212 et 393.

28 R. Rapin, Les Réflexions sur la poétique de ce temps et sur les ouvrages des poètes anciens et modernes, éd. E. T. Dubois, Genève / Paris, Droz / Minard, 1970, p. 103. 
plaisir lorsque l'on renouvelle [...] les plaies que le péché nous a faites ${ }^{29}$ ». De toute évidence, c'est à ce consentement dégradant que renvoie l'effémination. Et l'on retrouve la condamnation de ce cercle vicieux dans les textes contre l'opéra qui montrent comment le théâtre lyrique force les spectateurs à entretenir passionnément leurs «piq[ûres] »: «Les hommes sensibles à leurs appas [ceux des actrices de l'Opéra] échauffent par leurs applaudissements et leurs flatteries ces serpents à mesure qu'ils les piquent ${ }^{30}$. »

Ainsi, avec l'effémination, la femme semble convoquée comme principe de diminution de l'homme-masculin, principe qui, en tant que tel, entame l'homme-humain. L'effémination a donc partie liée avec une sorte de déshumanisation, comme on l'entend dans cette phrase de Sénèque reprise par Lamy : « [je m'] en retournai[s] [des spectacles] non seulement plus avare, plus ambitieux, plus amateur des plaisirs et du luxe : mais encore plus cruel et moins homme "parce que, dit-il, j'ai été avec des hommes"31». En traduisant inhumanior par «moins homme », il semble que Lamy fasse communiquer la corruption de l'humanité, qui fonde le paradoxe sénéquien, avec la déchéance de la virilité vertueuse, qui s'entend alors à la fois par excès ("plus cruel ») et par amoindrissement (« moins homme »).

\section{Effets féminins du genre théâtral : séduction, fascination}

Ce n'est donc pas que l'effémination soit sans rapport avec les femmes, c'est qu'avec l'effémination comme motif de haine du théâtre se dit un rapport du théâtre au féminin qui ne dépend pas de la présence des femmes - un rapport d'homologie structurelle. Le théâtre, en effet, est semblable aux femmes, disent les textes de condamnation. Semblables à elles, c'est-à-dire d'abord séducteur. L'oratorien Jean-François Senault, examinant dans quelle mesure les spectacles peuvent servir à « relever l'éclat » des princes, peint « la Comédie » comme une femme qui « ne cherche qu'à plaire » :

La Comédie est le plus charmant de tous les divertissements ; elle ne cherche qu'à plaire à ceux qui l'écoutent; elle se sert de la douceur des vers, de la beauté des expressions, de la richesse des figures, de la pompe du Théâtre, des habits, des gestes et de la voix des acteurs; elle enchante tout à la fois les yeux et les oreilles; et pour enlever l'homme tout entier, elle essaye de séduire son esprit après qu'elle a charmé tous ses sens. Il faut être de bronze ou de marbre pour résister à tant d'appas, et j'avoue que les plus grands saints auraient peine à conserver leur liberté au milieu de tant d'agréables tentations. ${ }^{32}$

29 B. Lamy, Nouvelles réflexions sur l'art poétique, Paris, A. Pralard, 1678, p. 241.

30 L.-F. Ladvocat, op. cit., p. 77.

31 B. Lamy, op. cit., p. 225. La citation de Sénèque provient des Lettres à Lucilius, VII, 3.

32 J.-Fr. Senault, Le Monarque ou les Devoirs du Souverain, Paris, P. Le Petit, 1662, p. 231-232. 
Le théâtre séduit, comme les femmes. Et, comme elles, il n'a d'« appas » que décevants. On sait comment, dans les querelles sur la moralité du théâtre de cette période, les arguments du traité de Tertullien sur la «toilette des femmes ${ }^{33}$ " sont conjugués à ceux du De spectaculis. La Décision faite en Sorbonne touchant la Comédie, par exemple, rapproche les effets de l'opéra de ceux de la vue d'une «femme [...] parée ${ }^{34} »$. Parmi ces «par[ures]» trompeuses, celles du langages sont particulièrement incriminées. À ce sujet, le terme de cajolerie employé par Voisin est intéressant, car il désigne les paroles exagérément douces n'ayant pour but que d'obtenir du beau 35 (la « cage » de la « cajolerie » est celle des oiseaux qu'on incite à chanter par imitation). C'est la beauté miroitante et vaine de la forme qui attire au théâtre, comme le dit aussi Lamy accusant les poètes de «donne[r] un tour à ce qu'ils disent qui n'est point ordinaire, et qui nous enchante ${ }^{36}$ ». On reconnaît là le scénario de la fascination, dont le terme enchantement est alors un synonyme: le théâtre «fai[t] paraître beau ce qui est laid $^{37} »$.

Or ce scénario est sexué : ce sont d'abord les femmes qui fascinent, comme le montrent les images inquiétantes ou ridicules qui se glissent souvent chez Furetière: «On ne peut pas avoir tant d'amour pour cette laide sans fascination $^{38}$. » En cela, l'allégorie présente dans le violent réquisitoire contre le théâtre de Molière signé Rochemont n'a rien pour surprendre :

Ce qui fait rire en sa bouche [de Molière] fait souvent pitié sur le papier, et l'on peut dire que ses comédies ressemblent à ces femmes qui font peur en déshabillé et qui ne laissent pas de plaire quand elles sont ajustées. ${ }^{39}$

Le même présupposé motive le rapprochement entre les serpents - scitale ou basilic - et les comédiennes, ce qui permet à Voisin de suggérer par un glissement la nature féminine du genre théâtral : "Et il me semble que non seulement toutes les Comédiennes bien parées, et richement vêtues, mais encore toutes les Comédies ressemblent ce serpent ${ }^{40}$. » Enfin, si la référence au féminin fonctionne si bien pour dénoncer la fascination qui se produit au théâtre, c'est qu'en impliquant l'idée d'une dépendance des femmes à l'égard de leur corps, elle permet de faire entendre que l'outrage tient non seulement au mensonge, mais à l'usurpation de la place du vrai bien, c'est-à-dire à l'idolâtrie. Voisin cite

33 Tertullien, La Toilette des femmes (De cultu feminarum), Paris, Les Éditions du Cerf, 1971.

34 L. Pégurier, Décision faite en Sorbonne touchant la comédie, avec une réfutation des sentiments relâches d'un nouveau théologien sur le même sujet, par M. l'abbé L. P., Paris, J.-B. Coignard, 1694.

35 A. Furetière, Dictionnaire universel, La Haye / Rotterdam, Leers, 1690, art. « Cageoller ».

36 B. Lamy, op. cit., p. 3.

37 A. Furetière, op. cit., art. « Fasciner».

38 Ibid., art. « Fascination ».

39 B. A. Sr. D. R., Observations sur une comédie de Molière intitulée le Festin de Pierre, dans Molière, Euvres complètes, éd. G. Couton, Paris, Gallimard, 1971, t. II, p. 1200.

40 J. de Voisin, op. cit., p. 408. 
saint Jean Chrysostome pour mettre en scène cette substitution vicieuse : «N'avez-vous point horreur d'entendre les paroles impudiques d'une Comédienne des mêmes oreilles que vous entendez les paroles d'un Prophète, qui vous introduit dans les mystères de l'Écriture ${ }^{41}$ ? » À la place de la bonne parole, du logos donné par Dieu, une «impudique» voix féminine, phônè entachée de passions; à la place du vrai bien, les « fausses beautés » du théâtre.

\section{Quand l'autre du féminin sert à dire l'altération de l'identité}

On voit que les rigoristes, en convoquant le féminin, greffent l'association chrétienne de la femme avec le péché sur la condamnation platonicienne de l'imitation comme altération ${ }^{42}$. Il est d'ailleurs frappant que Bossuet, lorsqu'il reprend la condamnation du théâtre par Platon, ne retienne, parmi les objets dont l'imitation avilit, que ce qui concerne les femmes :

Ge fut aussi à Platon une des raisons de condamner le théâtre en général, parce que la coutume régulièrement ne permettant pas d'y produire les femmes, leurs personnages étaient représentés par des hommes qui devaient par conséquent, non seulement prendre l'habit et la figure, mais encore exprimer les cris, les emportements et les faiblesses de ce sexe : ce que ce philosophe trouvait si indigne, qu'il ne lui eût fallu que cette raison pour condamner la comédie. ${ }^{43}$

En opérant cette sélection, Bossuet fait de l'imitation des femmes la dégradation qui régit toutes les autres. Et à la différence de Platon, qui détaillait des situations pour illustrer des risques spécifiques et occasionnels, il motive sa condamnation par ce qu'il présente comme des traits généraux et permanents : « les cris, les emportements et les faiblesses de ce sexe », comme si « ce sexe » se caractérisait par une identité instable, une propension à sortir de soi ; comme s'il n'était pas seulement ce qu'il ne faut pas devenir quand on est un homme, mais le fait de devenir autre. Cela explique sans doute la répétition de l'interdit du travestissement dans les textes de cette période alors même qu'il est en réalité très peu pratiqué sur la scène française ${ }^{44}$.

Lorsqu'elle sert à exprimer la haine du théâtre, la référence au féminin paraît donc avoir pour fonction de signifier l'autre absolu, le toujours autre, même pour soi, notamment parce qu'on considère la femme comme aliénée par ses passions. Le succès, dans ces textes rigoristes à la fin du siècle, de la reformulation de l'axiome de saint Matthieu sur la pensée d'adultère par saint Cyprien de

41 Ibid., p. 90.

42 Platon, La République, 396a, éd. R. Baccou, Paris, GF-Flammarion, 1966, p. 147.

43 J.-B. Bossuet, Maximes et réflexions sur la Comédie, éd. cit., p. 56.

44 Il existe certes de nombreuses intrigues fondées sur le travestissement mais, hors de la fiction, l'incarnation d'un rôle par un comédien de l'autre sexe est très rare. Elle ne concerne que certains rôles féminins : vieilles femmes souvent ridicules, nourrices, personnages merveilleux inquiétants. 
Carthage est en cela particulièrement éclairant. Le sémantisme et l'étymologie du mot adultère cristallisent déjà cette idée de devenir autre ${ }^{45}$, et les changements qu'implique la reformulation ne laissent aucun doute sur le caractère incontournable de la référence au féminin pour parfaire le raisonnement. Chez saint Cyprien de Carthage, l'axiome de Matthieu qui vise le péché d'intention («Quiconque regarde une femme avec convoitise a déjà, dans son cœur, commis l'adultère avec elle ${ }^{46}$ ») devient : «L'adultère s'apprend en le voyant et [...] une femme mariée, venue pudique, peut-être, au spectacle, s'en retourne du spectacle impudique ${ }^{47}$. » On comprend l'élément commun : la culpabilité liée au simple fait de voir. Mais en passant dans le domaine du théâtre, l'adultère, de comportement coupable, est devenu sujet représenté sur la scène, provoquant par le simple fait d'être donné à voir une culpabilité bien réelle, celle des femmes, qui n'étaient chez saint Matthieu que l'objet de la tentation. On a donc un redoublement en une sorte de mise en abyme: le théâtre fait commettre l'adultère en le donnant à voir. Mais on sait qu'en un sens, la nature de ce qui est représenté n'importe pas. C'est par le simple fait d'inciter à se projeter hors de soi, quel que soit le scénario qui supporte cette projection, que le théâtre altère, d'une manière que seule la référence au féminin semble à même d'exprimer.

On comprend alors l'importance du motif de la prostitution. Pour dire le sacrilège que constitue l'exposition publique des vices et des faux désirs (Voisin, citant Jean de Salisbury, accuse la voix des comédiens de «prostitue[r] [...] les oreilles, et le cœur à la vanitét8 »), seule convient l'idée qu'on se fait de la prédisposition des femmes à se donner, à circuler publiquement; et seul convient l'abandon féminin pour dire le «transpor[t]», l'«abando[n]» de l'esprit qui se produit au théâtre. Nicole affirme ainsi que «l'esprit [...] étant [à la Comédie] transporté et tout hors de soi, au lieu de corriger [les mauvais] sentiments [en l'occurrence, l'honneur et la vengeance] s'y abandonne sans résistance 49 ». Si bien qu'au plus loin, en un sens, de l'implication de femmes réelles lors de la séance théâtrale, on retrouve la condamnation la plus concrète du théâtre comme lieu de débauche.

\section{Le genre du genre?}

On le voit, les modalités d'incrimination des femmes sont diverses, et dessinent un «féminin» plein de bizarreries logiques: les femmes sont incriminées comme cibles trop dociles du spectacle, mais aussi comme moteur

45 Adulterare, formé sur alterare signifie au sens propre : " rendre autre », « falsifier ».

46 Matthieu, V, 28, Traduction œcuménique de la Bible, Paris / Villiers-le-Bel, Les Éditions du Cerf / Société Biblique Française, 1998.

47 Cyprien de Carthage, À Donat, n. 8, 1. 174, éd. J. Molager, Paris, Les Éditions du Cerf, 1982 , p. 95 ; cité par L. Thirouin, op. cit., p. 125.

48 J. de Voisin, op. cit., p. 120.

49 P. Nicole, op. cit., § XVIII, p. 74. 
de sa nocivité, du fait de leurs attentes qui programment son fonctionnement; elles sont victimes et coupables, et, par là, mises sur le même plan que les hommes auxquels elles sont supposées inspirer des désirs vicieux; elles sont en cause dans la condamnation du travestissement alors même que celui-ci repose sur l'élimination de leur présence. Il y a donc bien sûr de la figure : c'est par métonymie, métaphore, allégorie, que les femmes servent à dire la haine du théâtre, et ce sont toutes ces figures qui font du théâtre un genre pour lequel la présence des femmes ne peut être simplement circonstancielle, un genre qu'on ne peut "sépare $[\mathrm{r}]$ » des femmes, même "par l'imagination », pour reprendre la formule de Nicole dont nous avons montré la réversibilité.

Finalement, où sont les femmes ? Si elles adhèrent de telle manière au théâtre haï, s'il y a figure à ce point, c'est peut-être qu'il ne faut pas les chercher trop concrètement dans cette haine du théâtre. Ce paradoxe, les travaux pionniers de l'anthropologue Mary Douglas sur le concept de «souillure » nous aident à le penser :

Il existe des croyances, écrit-elle, selon lesquelles chacun des sexes constitue un danger pour l'autre quand ils entrent en contact par le truchement des fluides sexuels. Chez certains peuples, le contact n'est dangereux que pour l'un des sexes. C'est généralement la femme qui met l'homme en danger, quoique l'inverse puisse être vrai aussi. Dans le domaine sexuel, ces notions de danger sont l'expression d'une symétrie ou d'une hiérarchie. Il est peu probable qu'elles expriment quelque aspect des rapports réels entre les sexes. À mon avis, il faut les considérer comme l'expression symbolique des relations entre différents éléments de la société, comme le reflet d'une organisation hiérarchique ou symétrique qui vaut pour l'ensemble du système social. 50

Si l'on suit cette conception, le recours au féminin serait donc moins l'expression d'un acharnement à l'encontre des femmes réelles qu'un moyen de stigmatiser la capacité du théâtre à troubler l'organisation hiérarchique et symbolique de la société. On voit la fécondité d'une telle perspective : considérer les figures féminines de la haine du théâtre permet alors d'explorer le potentiel de désordre autant que d'expérimentation d'un ordre nouveau prêté au théâtre par ses détracteurs - programme passionnant qui s'inscrit dans la lignée des travaux d'Hélène Merlin-Kajman, montrant comment le théâtre, en prise sur les bouleversements occasionnés par le régime absolutiste, contribue alors à défaire et à redéfinir la notion de "public», et comment, en tant que tel, il pose problème et forme un paradigme avec d'autres «lieux », au nombre desquels la cour et les femmes ${ }^{51}$; ou encore dans la lignée des travaux de Mitchell Greenberg :

50 M. Douglas, Purity and Danger: an Analysis of Concepts of Pollution and Taboo, Londres, Routledge \& Kegan Paul, 1966. Trad. fr. : De la souillure. Essai sur les notions de pollution et de tabou, Paris, La Découverte, 2001, p. 25.

51 H. Merlin-Kajman, L'Absolutisme dans les Lettres et la théorie des deux corps. Passions et Politique, Paris, H. Champion, 2000 ; id., « Les troubles du masculin en France au XVII ${ }^{e}$ siècle », art. cit. 
Le théâtre ne se contente pas de représenter la réalité sociale de l'époque, il la remet en question. Dans un certain sens, on pourrait dire que le théâtre est, $[\ldots]$ fondamentalement ambivalent, tout à la fois « ordre et désordre ».52

Mais il n'est pas sûr qu'en faisant cela on doive subsumer la question du féminin sous celle du théâtre. On peut au contraire considérer comme spécifique la définition qui est donnée des femmes au travers de l'expression de cette haine - spécifique et ancrée historiquement, comme le montrent non seulement les infléchissements repérés dans la reprise de certains motifs, mais aussi les textes contemporains qui, de manière inversement symétrique, recourent au théâtre pour caractériser les défauts des femmes. Deux exemples rapides suffiront: Fénelon affirmant que celles-ci « ont un naturel souple pour jouer facilement toutes sortes de comédies ${ }^{53}$ »; et Perrault, lorsqu'il doit, au début de son Apologie des femmes, concéder le vice d'un petit pourcentage d'entre elles: «Pour six qui sans cervelle avec un peu d'appas, / Feront de tous côtés du bruit et du fracas, / Par leur danse, leur jeu, leurs folles mascarades ${ }^{54} »$.

Ce serait donc parce que le féminin est conçu comme dépendant du théâtre qu'il est convoqué pour stigmatiser le théâtre - conçu comme dépendant, voire conçu comme théâtre lui-même ${ }^{55}$. Mais alors, n'est-ce pas que ces figures du féminin comme théâtre sont en fait l'emblème du genre, c'est-à-dire qu'elles renvoient non pas à une identité sexuelle biologiquement fixée, mais à une identité moralement, socialement, culturellement construite? Cela reviendrait à affirmer qu'en haïssant le théâtre, les rigoristes de la seconde moitié du XVIIe siècle haïssent le genre, autrement dit que le théâtre est pour eux le genre du genre. Il est d'autant plus tentant de le penser que le récent ouvrage d'Anne-Emmanuelle Berger a bien montré ce que l'élaboration du concept de genre, dès les années 1950, doit au théâtre - au théâtre comme dispositif où s'accomplit dans la performance ce qui n'a pas d'essence première, où jeu et costume sont moteurs de vérité, mais surtout au théâtre comme dynamique érotisante du fait même de sa dimension construite et de sa tension toujours inaccomplie vers un idéal ${ }^{56}$. L'hypothèse que nous faisons est que c'est ce possible fonctionnement du théâtre, bien repéré par ses détracteurs, qui motive leur haine.

Il est moins évident de démêler les implications de cet échange de qualités entre «femme » et «théâtre » au niveau de la représentation du féminin. Selon

52 M. Greenberg, "Early modern" : un concept problématique ? », Transitions, n 7, en ligne : http://www.mouvement-transitions.fr/intensites/transition/sommaire-general-de-transition/ 491-n-7-m-greenberg-early-modern-un-concept-problematique.html (consulté le 5 avril 2019).

53 Fr. de Fénelon, De l'éducation des filles, chap. IX, Euvres, éd. J. Le Brun, Paris, Gallimard, «Bibliothèque de la Pléiade », 1983-1997, t. I, p. 146.

54 Ch. Perrault, Apologie des femmes, Paris, J.-B. Coignard, 1694, p. 5.

55 On pense nécessairement à la théorie qu'en fera Jacques Lacan, reprenant à Joan Rivière le terme de mascarade (Le Séminaire de Jacques Lacan. Livre V, Paris, Éditions du Seuil, 1998, p. 251).

56 A.-E. Berger, Le Grand Théâtre du genre. Identités, Sexualités et Féminisme en "Amérique ", Paris, Belin, 2013. 
les célèbres travaux de Thomas Laqueur ${ }^{57}$, la période voit le déclin d'une pensée du genre héritée de l'Antiquité - homme et femme ne présentant qu'une différence de degré autour d'un modèle unique - au profit de l'idée de deux sexes bien distincts en nature. Serait-ce cette tendance que l'on reconnaît dans les réactions virulentes de cette fin du XVIIe siècle: le besoin d'arrimer le féminin à une naturalité pour le séparer essentiellement du masculin ? Mais il est difficile d'élucider la position des rigoristes à ce sujet sans la simplifier exagérément, plus encore de la situer dans un mouvement de progression des idées - n'avons-nous pas quelque réticence à imaginer les ennemis du théâtre en pionniers d'une nouvelle pensée des rapports entre masculin et féminin ?

Il faut donc admettre qu'avec de telles questions est aussi en jeu notre idée de ce qui est réactionnaire et de ce qui est progressiste. Aussi notre conclusion serat-elle prudente. Ce que nous pouvons affirmer, c'est que la haine du théâtre, dans une certaine mesure, épargne les femmes réelles pour se nourrir d'inquiétudes anthropologiques, sociales, morales, religieuses, qui sont en rapport avec le caractère construit des valeurs et des identités. Le reste n'est qu'incitation méthodologique : pour bien comprendre cela, il faut résister aux partages binaires ainsi qu'aux réflexes téléologiques plus ou moins conscients qui posent comme nécessairement émancipateur l'amour du théâtre, du plaisir et du féminin - et faire place, plutôt, aux contradictions : entre diverses pensées du genre (continuum asservissant versus refus du déterminisme biologique), entre hostilité butée et compréhension aiguë d'un certain fonctionnement du théâtre chez les détracteurs, et enfin - ce n'est pas le moins intrigant - entre l'objet que ceux-ci condamnent et le théâtre de figures dressé au service de cette condamnation.

\author{
Sarah Nancy \\ Université Sorbonne Nouvelle - Paris 3
}




\section{littératures classiques}

\section{Sommaire}

François Lecercle et Clotilde Thouret Introduction

Le théâtre, lieu démoniaque et art trompeur

Marie-Hélène Goursolas

La notion d'idolâtrie dans la polémique contre le théâtre en France et en Angleterre aux $x v l^{e}$ et $x V I^{e}$ siècles: enjeux d'un argument récurrent

\section{Enrica Zanin}

La vraisemblance: un argument contre le théâtre?

Marie Saint-Martin

Haine du théâtre et démonologie: de la possession démoniaque à la catharsis

\section{Florence d'Artois}

«Y Agustino, capitán de todos»? Paradoxes de l'augustinisme dans les controverses sur le théâtre en Espagne

Le spectacle, ou le règne du sensible

\section{Françoise Decroisette}

La peur du corps dans les textes sur le théâtre des zanni aux $\left.x \mathrm{~V}\right|^{\mathrm{e}}$ et $\mathrm{XVI} \mathrm{I}^{\mathrm{e}}$ siècles

\section{Andrea Fabiano}

Haine du théâtre, haine des femmes: fascination et censure dans la réflexion théorique et dans la pratique théâtrale italiennes au XVIII siècle

Véronique Lochert

«Regarder hardiment et avec plaisir» ou «détourner les yeux»? Les dangers du théâtre pour les spectatrices (France-Angleterre, $x \mathrm{xl}^{\mathrm{e}}-\mathrm{xVII} \mathrm{e}^{\mathrm{e}}$ siècles)

\begin{abstract}
Sarah Nancy
De la condamnation de la mixité à la crainte de l'altération. Où sont les femmes dans la haine du thêâtre?
\end{abstract}

\section{Sylviane Léoni}

Fiction et fonction du regard dans le discours antithéâtral

\section{Marion Lafouge}

Avatars et métamorphoses d'une nouvelle querelle coloriste. Le paradigme pictural dans les discours et débats sur l'opéra

\section{Anthropologies du spectateur}

\section{Ellen MacKay}

La figure du public dans l'imaginaire théâtrophobe anglais de la première modernité

\section{Bruna Filippi}

La tolérance comme masque de la haine du théâtre (Italie, $x v \|^{e}$ siècle)

\section{Logan J. Connors}

«Sur la superficie de notre cœur » : de la condamnation du théâtre à une science théâtrale de l'homme (16941719)

\section{Larry Norman}

La théâtrophobie et la «nouvelle philosophie» moderne: le cas de Jean Terrasson

\section{Résumés}

Collection de rééditions de textes [rares] du XVII siècle

Bibliothèque des Littératures Classiques

Numéros parus

\section{PUM}

universitaires
PRESSES UNIVERSITAIRES DU MIDI

UNIVERSITÉ TOULOUSE - JEAN JAURÈS

pum.univ-tse2.fr

PRIX : $25 €$

\section{SORBONNE SORBONNE
UNIVERSITE 0 Observatoire}

